\title{
Relación entre suelos y estructura del bosque en la Amazonía colombiana
}

\section{Bayron R. Calle-Rendón ${ }^{1}$, Flavio Moreno ${ }^{2}$ \& Dairon Cárdenas-López ${ }^{3}$}

1. Ingeniero Forestal, Universidad Nacional de Colombia Sede Medellín, Apartado Aéreo 568, Medellín, Colombia; brcalle@unal.edu.co

2. Grupo de Investigación en Ecología y Silvicultura de Especies Forestales Tropicales, Universidad Nacional de Colombia Sede Medellín, Apartado Aéreo 568, Medellín,Colombia; fhmoreno@unal.edu.co

3. Instituto Amazónico de Investigaciones Científicas SINCHI, Apartado Aéreo 034174, Bogotá D.C., Colombia; dcardenas@sinchi.org.co

\section{Recibido 31-VIII-2010. Corregido 10-XII-2010. Aceptado 19-I-2011.}

\begin{abstract}
Soil and forest structure in the Colombian Amazon. Forests structural differences could result of environmental variations at different scales. Because soils are an important component of plant's environment, it is possible that edaphic and structural variables are associated and that, in consequence, spatial autocorrelation occurs. This paper aims to answer two questions: (1) are structural and edaphic variables associated at local scale in a terra firme forest of Colombian Amazonia? and (2) are these variables regionalized at the scale of work? To answer these questions we analyzed the data of a 6ha plot established in a terra firme forest of the Amacayacu National Park. Structural variables included basal area and density of large trees (diameter $\geq 10 \mathrm{~cm})(\mathrm{Gdos}$ and $\mathrm{Ndos}$ ), basal area and density of understory individuals (diameter $<10 \mathrm{~cm}$ ) (Gsot and Nsot) and number of species of large trees (sp). Edaphic variables included were $\mathrm{pH}$, organic matter, $\mathrm{P}, \mathrm{Mg}, \mathrm{Ca}, \mathrm{K}, \mathrm{Al}$, sand, silt and clay. Structural and edaphic variables were reduced through a principal component analysis (PCA); then, the association between edaphic and structural components from PCA was evaluated by multiple regressions. The existence of regionalization of these variables was studied through isotropic variograms, and autocorrelated variables were spatially mapped. PCA found two significant components for structure, corresponding to the structure of large trees (G, Gdos, Ndos and sp) and of small trees (N, Nsot and Gsot), which explained $43.9 \%$ and $36.2 \%$ of total variance, respectively. Four components were identified for edaphic variables, which globally explained $81.9 \%$ of total variance and basically represent drainage and soil fertility. Regression analyses were significant $(\mathrm{p}<0.05)$ and showed that the structure of both large and small trees is associated with greater sand contents and low soil fertility, though they explained a low proportion of total variability $\left(\mathrm{R}^{2}\right.$ was $4.9 \%$ and $16.5 \%$ for the structure of large trees and small tress, respectively). Variables with spatial autocorrelation were the structure of small trees, Al, silt, and sand. Among them, Nsot and sand content showed similar patterns of spatial distribution inside the plot. Rev. Biol. Trop. 59 (3): 1307-1322. Epub 2011 September 01.
\end{abstract}

Key words: spatial autocorrelation, Amacayacu National Park, edaphic variables, basal area, tree density.

Aproximadamente la mitad de la superficie de bosques del mundo se encuentran en la zona tropical. De la totalidad de los bosques tropicales existentes el $47 \%$ están situados en África tropical, el sudeste asiático y algunas islas de Oceanía y el 52\% restante se localiza en los trópicos americanos (FAO 2009). Los bosques amazónicos representan la mayor extensión forestal del neotrópico y del planeta y son considerados como uno de los ecosistemas más importantes del mundo no sólo por su contribución a los ciclos mundiales de agua y carbono (Soares-Filho et al. 2006) sino también por su alta riqueza y complejidad ecológica (Hartshorm 2002).

El dinamismo de los bosques tropicales es el producto de un amplio rango de factores $\mathrm{y}$ procesos que ocurren a diferentes escalas 
espaciales y temporales; por ejemplo, la aparición de barreras físicas en grandes áreas que se han presentado en tiempos geológicos, o las variaciones en la intensidad de la luz que ocurren en unos pocos segundos y abarcan unos pocos metros cuadrados (Scatena 2002). A grandes escalas de paisaje, el tipo de suelo influencia fuertemente la vegetación; por ejemplo, el bosque amazónico que crece sobre arenas blancas (Varillal) difiere estructuralmente del que crece en tierra firme, pues el primero es un ecosistema de pequeños arbustos y árboles de estructura menos compleja y de menor diversidad que el bosque de tierra firme (Clark 2002). Entre tanto, a pequeñas escalas, la dinámica de claros y las variaciones topográficas crean microhábitats que cambian las condiciones ambientales y producen diferencias en el bosque (Svenning 1999); incluso, es posible que factores edáficos variados en pequeñas escalas distintos a la topografía provoquen diferencias en el bosque.

Algunos estudios han demostrado que existen asociaciones entre los componentes del suelo y algunas variables estructurales del bosque, comúnmente entre especies y tipos de suelo (Duivenvoorden 1996, Clark 2002, Duivenvoorden et al. 2005); sin embargo, son muy pocos los estudios que se han ocupado en evaluar las asociaciones entre el suelo y otras variables estructurales diferentes del número de especies: por ejemplo, Clark \& Clark (2000) encontraron que el área basal y la densidad de individuos era menor en suelos fértiles de Costa Rica. Similarmente, Duivenvoorden et al. (2005) reportaron que la densidad de individuos era menor en suelos de fertilidad relativamente alta en el noroccidente del Amazonas. Estas variaciones estructurales en áreas relativamente pequeñas evidencian la asociación entre la estructura del bosque y factores externos del ambiente, entre ellos los factores edáficos. Ello quiere decir que las comunidades de plantas o algunas de sus variables estructurales responden a las condiciones ambientales del sitio, por lo que tales variables podrían ser regionalizadas (es decir, que presentan autocorrelación espacial); de hecho, algunos estudios sugieren que las comunidades de plantas se estructuran en el espacio en respuesta a factores abióticos (Guo et al. 2002, Schwarz et al. 2003).

Según la teoría del nicho, la composición de la comunidad cambia como resultado de la respuesta adaptativa de las especies a los gradientes ambientales (Gilbert \& Lechowics 2004), entre ellos las diferencias edáficas. Por eso muchos procesos ecológicos resultan restringidos por las condiciones ambientales de manera que varían en el tiempo y en el espacio (Wagner \& Fortin 2005), por lo cual, tales procesos pueden depender, al menos parcialmente, de la variabilidad espacial del ambiente. De este modo, la heterogeneidad espacial del medio físico y de los recursos provoca la aparición de regionalización en las poblaciones y comunidades (Camarero \& Gutiérrez 1999). De ahí la necesidad de involucrar técnicas como la geoestadística en investigaciones ecológicas, las cuales se han usado en diversos estudios entre los que se encuentran análisis de ecosistemas estuarinos (Giraldo et al. 2000), distribución de la biomasa de raíces gruesas en bosques primarios (Sierra 2001) y distribución de nutrientes en el suelo (Jackson \& Caldwell 1993).

En el presente estudio se evaluó la posible relación entre los factores edáficos y estructurales a escala local, en una parcela de seis hectáreas de bosque de tierra firme en el Parque Nacional Natural Amacayacu de la Amazonía colombiana. Para ello se pretende dar respuesta a los siguientes interrogantes: (1) ¿Existe asociación entre las variables edáficas y estructurales del bosque? y (2) ¿Son las variables edáficas y estructurales regionalizadas a la escala del trabajo?

Por consiguiente se estima que la respuesta a estas preguntas permita la identificación de algunas características específicas del ambiente necesarias para el desarrollo de determinados rasgos estructurales del bosque, lo cual permite obtener un conocimiento ecológico más concreto de las asociaciones entre la vegetación arbórea y el medio abiótico. Adicionalmente, se confía en su utilidad para plantear estrategias 
dirigidas a la conservación y el manejo de los bosques tropicales.

\section{MATERIALES Y MÉTODOS}

Área de estudio: El Parque Nacional Natural Amacayacu (PNNA) está ubicado en el trapecio amazónico colombiano al sur del país ( $3^{\circ} 49^{\prime} \mathrm{S}$ y $70^{\circ} 19^{\prime} \mathrm{W}$ ). La temperatura media anual es de $25^{\circ} \mathrm{C}$, la humedad relativa es bastante alta con un promedio anual de $86 \%$ y la precipitación media es de $3023 \mathrm{~mm}$ por año con una distribución unimodal concentrada entre los meses de octubre y mayo, y con un período menos lluvioso entre junio y septiembre. Las principales unidades geológicas son sedimentos de la planicie del Terciario, sedimentos plio-pleistocénicos y aluviones recientes del Cuaternario. El PNNA presenta un relieve colinado con elevaciones que oscilan entre 80 y 200m (Rudas \& Prieto 1998). Los suelos se caracterizan por un nivel bajo de fertilidad, alta acidez y baja saturación de bases, en los cuales predominan minerales pobres en nutrientes como caolinita y cuarzo (Chamorro 1989).

Variables estructurales del bosque: Así pues, se dividió una parcela de 6ha (120x500m) en 150 sub-parcelas de $20 \times 20 \mathrm{~m}$; el centro de cada una se tomó como referencia para establecer sub-parcelas de 0.01 ha $(10 \mathrm{x} 10 \mathrm{~m})$. Se seleccionó el extremo noroccidental de la parcela para incluir 9 sub-parcelas de 20x20m y cada una se dividió en 4 sub-parcelas para formar 36 sub-parcelas auxiliares de 0.01 ha $(10 \times 10 \mathrm{~m})$ (Fig. 1) y asegurar una distancia mínima de $10 \mathrm{~m}$ entre sub-parcelas. Entonces, fue posible obtener una base de datos con 150 sub-parcelas denominada base de datos uno (BD-1) y otra con 177 sub-parcelas denominada base de datos dos (BD-2) en la que están incluidas las sub-parcelas auxiliares. En todas las sub-parcelas se midieron los árboles con DAP (diámetro a la altura del pecho a $1.3 \mathrm{~m}$ desde el suelo) mayor o igual a un centímetro y se distinguieron como individuos de sotobosque $(1 \mathrm{~cm} \leq \mathrm{DAP}<10 \mathrm{~cm})$ y de dosel $(\mathrm{DAP} \geq 10 \mathrm{~cm})$. Se determinaron variables estructurales como el número de especies (sp) para los árboles de dosel; el número total de individuos $(\mathrm{N})$, el número de individuos de sotobosque (Nsot) y de dosel (Ndos), el área basal total (G), además del área basal de individuos de sotobosque (Gsot) y de dosel (Gdos). La identificación de las colecciones botánicas se hizo en el Herbario Amazónico Colombiano (COAH).

Variables edáficas: De cada sub-parcela de $10 \times 10 \mathrm{~m}$ se tomaron tres muestras de suelo de 0 a $10 \mathrm{~cm}$ de profundidad del mismo volumen a una distancia de cinco metros entre cada una a lo largo del eje central norte-sur de la sub-parcela y se mezclaron para obtener una muestra compuesta. El proceso de preparación de las muestras de suelo realizado en el Laboratorio de Ecología y Conservación Ambiental (LECA) de la Universidad Nacional de Colombia Sede Medellín, incluyó el secado al aire, pulverización con rodillo, tamizado y mezcla, con el objeto de que la muestra analizada fuera representativa de la sub-parcela muestreada. Luego se determinó el contenido de $\mathrm{Ca}, \mathrm{Mg}$ y $\mathrm{K}$ por absorción atómica, el porcentaje de materia orgánica (MO) con la prueba de Walkley \& Black, el contenido de $\mathrm{P}$ mediante emisión de UV-VIS con espectrofotómetro, el pH con el método potenciométrico de suelo y la textura por el método de Bouyoucos. Los resultados de los análisis de suelos se anexaron a la BD-1.

Análisis de datos: Para reducir el número de variables edáficas y estructurales se realizaron dos análisis de componentes principales (ACP) con la BD-1 y para verificar si existía asociación entre ambos grupos de variables, se evaluó un conjunto de regresiones múltiples entre los valores de los vectores propios de los componentes estructurales en función de los valores de los vectores propios de los componentes edáficos en STATGRAPHICS Centurion XV (Versión 15.2.06).

Para el análisis espacial y explorar la regionalización de las variables edáficas y estructurales se construyeron variogramas isotrópicos y se tomó en cuenta la BD-1 y la BD-2 respectivamente. En ellos se analizaron algunos parámetros claves como el nugget o 


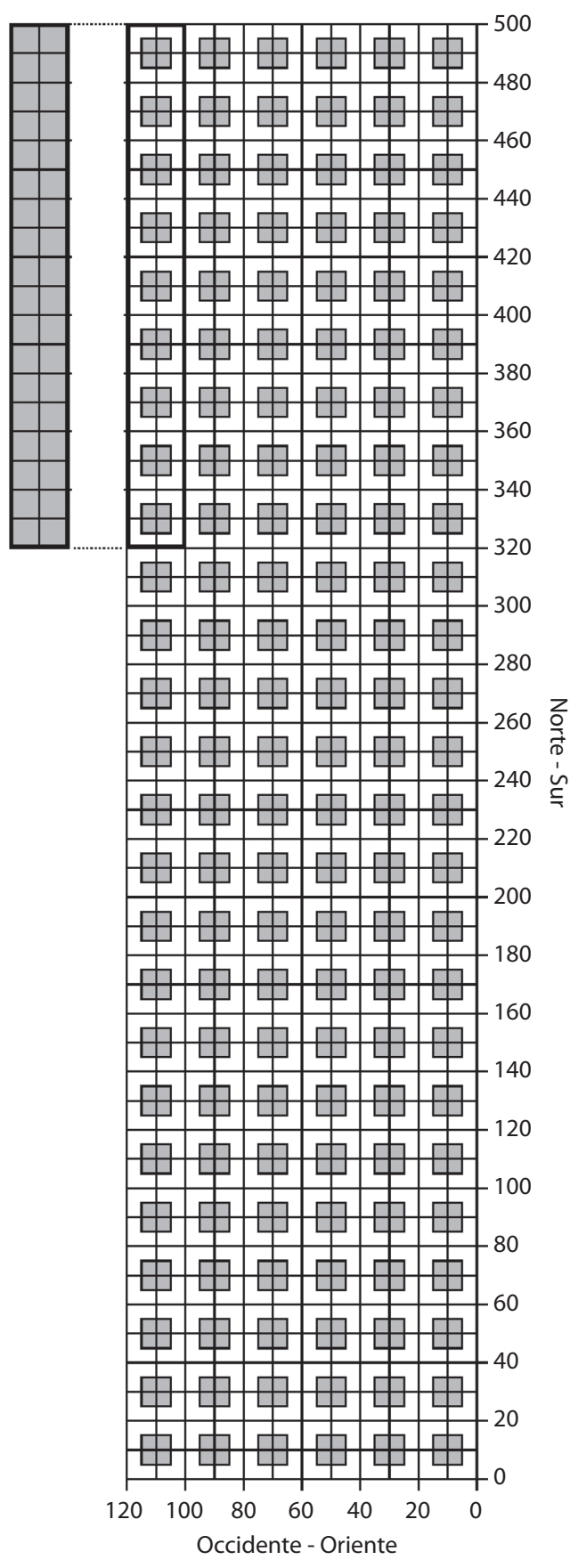

efecto pepita $\left(C_{0}\right)$, el sill o meseta $\left(C_{0}+C\right)$ y el rango $\left(A_{0}\right)$ (Fig. 2) y, mediante la técnica de interpolación Kriging (Gallardo 2006) se construyeron mapas de distribución espacial de toda la parcela para las variables que presentaron autocorrelación espacial. El análisis se realizó en el software $\mathrm{GS}^{+}$GAMMA DESIGN SOFTWARE, Versión 9.0 (Plainwell, Michigan USA).

\section{RESULTADOS}

Al área basal total aportaron más los individuos de dosel que los individuos de sotobosque ( 87.5 y $12.5 \%$, respectivamente); entre tanto, el número de individuos de sotobosque hizo el mayor aporte al número de individuos total $(88.13 \%)$ (Cuadro 1). Por otra parte, Barreto et al. (2010) encontraron en un estudio paralelo en cinco hectáreas de la parcela del PNNA 510 especies (las más abundantes fueron Eschweilera itayensis, Otoba parvifolia y Eshweilera coriacea) pertenecientes a 62 familias (las más abundantes fueron Lecythidaceae, Myristicaceae y Moraceae).

$\mathrm{El} \mathrm{pH}$ de todas las 150 muestras de suelo fue inferior de cinco (promedio de 4.31), por lo cual puede decirse que la totalidad de la parcela se caracteriza por tener suelos ácidos. El promedio de bases presentes en el suelo,

Fig. 1. Parcela de 6ha $(120 \times 500 \mathrm{~m})$ en el PNNA. Los números indican la distancia en metros y las zonas sombreadas corresponden a las 150 sub-parcelas de 0.01 ha (10x10m) dentro de las sub-parcelas de 20x20m (BD1). En la extremo noroccidental se seleccionaron nueve sub-parcelas de $20 \times 20 \mathrm{~m}$ y cada una se dividió en cuatro sub-parcelas de 0.01 ha $(10 \times 10 \mathrm{~m})$; estas conforman en total 36 sub-parcelas auxiliares para un total de 177 sub-parcelas (BD-2).

Fig. 1. Representation of the 6ha plot $(120 \times 500 \mathrm{~m})$ in the Amacayacu National Park. Numbers indicate the distance in meters and shaded areas correspond to the $1500.01 \mathrm{ha}(10 \mathrm{x} 10 \mathrm{~m})$ sub-plots inside the 20x20m sub-plots (BD-1). Nine 20x20m sub-plots were selected in the northwest corner and each one of them was divided into four 0.01 ha sub-plots $(10 \times 10 \mathrm{~m})$. Total number of sub-plots was 177 (BD-2) summing these auxiliary sub-plots. 


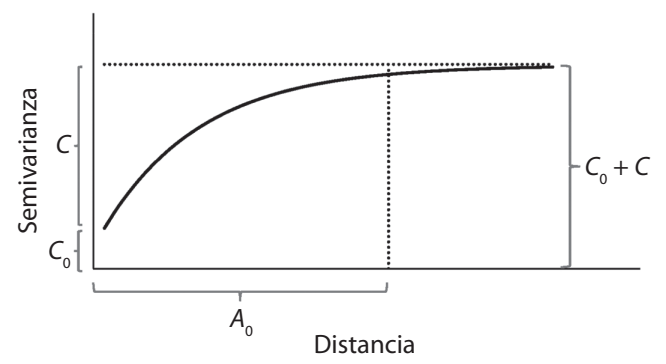

Fig. 2. Parámetros utilizados para el análisis de un variograma isotrópico. Nugget o efecto pepita $\left(C_{0}\right)$ : varianza no explicada por el modelo. Semivarianza $(C)$ : varianza explicada por el modelo. Sill o meseta $\left(C_{0}+C\right)$ : máxima varianza entre pares de puntos. Rango $\left(A_{0}\right)$ : distancia en la que variable presenta autocorrelación espacial y a partir de la cual es aleatoria.

Fig. 2. Parameters used for the analysis of an isotropic variogram. Nugget $\left(C_{0}\right)$ : variance unexplained by the model. Semivariance $(C)$ : variance explained by the model. Sill $\left(C_{0}+C\right)$ : maximum variance between pairs of points. Range $\left(A_{0}\right)$ : maximimum distance of spatial autocorrelation (beyond this distance samples are random).

correspondiente a la suma de las concentraciones de $\mathrm{Ca}, \mathrm{Mg}$ y $\mathrm{K}$ fue de $1.61 \mathrm{meq} / 100 \mathrm{~g}$ de suelo. El Al promedio fue de $0.68 \mathrm{meq} / 100 \mathrm{~g}$ de suelo. En cuanto a la textura, el porcentaje medio de limo superó la media de arena y arcilla con un valor de $45.79 \%$ sobre 30.12 y $24.08 \%$, respectivamente (Cuadro 2); la textura resultante del suelo es franco arcilloso.
La reducción del número de variables estructurales del bosque lograda mediante el ACP determinó claramente dos componentes estadísticamente significativos; el primero de ellos parece explicar lo concerniente a la estructura de los individuos de dosel $\left(\mathrm{Cl}_{\text {est }}\right)$ con una varianza explicada del $43.90 \%$ y el segundo explica la estructura de los individuos de sotobosque $\left(\mathrm{C} 2{ }_{\text {est }}\right)$ con una varianza del $36.23 \%$ (Cuadro 3).

El ACP realizado para las variables edáficas determinó cuatro componentes principales estadísticamente significativos los cuales explican la mayor variabilidad de los datos con una varianza acumulada del $81.93 \%$. En este análisis se omitieron los contenidos individuales de $\mathrm{Ca}, \mathrm{Mg}$ y $\mathrm{K}$, y se optó por incluir la suma de estas tres variables (bases). El primer componente aporta el $27.61 \%$ de la varianza total, el segundo el $25.23 \%$, el tercero el $16.31 \%$ y el cuarto el $12.78 \%$. Al primer componente $\left(\mathrm{C} 1_{\text {edaf }}\right)$ se encuentran asociados las bases, el pH y los contenidos de limo y arcilla. El segundo componente $(\mathrm{C} 2$ edaf $)$ está representado principalmente por el $\mathrm{Al}$, el $\mathrm{pH}$, la arena y el limo y en él se observa claramente como el pH tiene alta correlación (pero negativa) con el $\mathrm{Al}$, mientras que la arena se correlaciona con el contenido de limo. En el tercer componente $\left(\mathrm{C} 3_{\text {edaf }}\right)$ las variables de mayor peso son el contenido de arena y el fósforo, aunque estas están

CUADRO 1

Variables estructurales del bosque en una parcela de 6ha (120x500m) en el PNNA usando la BD-2

TABLE 1

Structural variables in a 6ha plot $(120 \times 500 \mathrm{~m})$ in the Amacayacu National Park using the BD-2

$\begin{array}{ccccccccc} & \text { G } & \text { Gsot } & \text { Gdos } & \mathrm{N} & \text { Nsot } & \text { Ndos } & \text { sp/0.01ha } \\ & & \mathrm{m}^{2} / 0.01 \mathrm{ha} & & & \text { Individuos/0.01ha } & & 5.24 \\ \bar{X} & 0.32 & 0.04 & 0.28 & 48.89 & 43.09 & 5.80 & 2.08 \\ S & 0.20 & 0.01 & 0.20 & 12.62 & 12.39 & 2.28 & 11.00 \\ \text { Max } & 1.19 & 0.08 & 1.16 & 89.00 & 87.00 & 13.00 & 1.00 \\ \text { Min } & 0.05 & 0.01 & 0.01 & 17.00 & 16.00 & 1.00 & 1.00\end{array}$

Media $(\bar{X} \bar{X})$, desviación estándar (S) y valor máximo (Max) y mínimo (Min) de cada una de las variables estructurales para las 177 sub-parcelas de $10 \times 10 \mathrm{~m}$.

Average $(\bar{X} \bar{X})$, standard deviation (S), maximum (Max) and minimum (Min) of each structural variable for 177 sub-plots of $10 \times 10 \mathrm{~m}$. 
CUADRO 2

Variables edáficas en una parcela de 6ha (120x500m) en el PNNA usando la BD-1

TABLE 2

Edaphic variables in a 6ha plot (120x500m) in the Amacayacu National Park using the BD-1

\begin{tabular}{cccccccccccccc} 
& & & & $\mathrm{Al}$ & $\mathrm{Ca}$ & $\mathrm{Mg}$ & $\mathrm{K}$ & Bases & Arena & Limo & Arcilla \\
& $\mathrm{pH}$ & $\mathrm{MO}(\%)$ & $\mathrm{P}(\mathrm{ppm})$ & \multicolumn{9}{c}{ meq/100g de suelo } \\
$\bar{X}$ & 4.31 & 3.04 & 4.44 & 0.68 & 0.84 & 0.51 & 0.26 & 1.61 & 30.12 & 45.79 & 24.08 \\
$S$ & 0.27 & 0.73 & 2.05 & 0.17 & 0.81 & 0.32 & 0.11 & 1.08 & 6.24 & 7.39 & 5.59 \\
$\operatorname{Max}$ & 4.88 & 6.12 & 15.05 & 1.00 & 6.22 & 2.44 & 0.56 & 7.73 & 48.00 & 64.00 & 40.00 \\
Min & 3.75 & 1.36 & 0.35 & 0.30 & 0.12 & 0.17 & 0.10 & 0.47 & 16.00 & 30.00 & 2.00
\end{tabular}

Media $(\bar{X} \bar{X})$, desviación estándar (S) y valor máximo (Max) y mínimo (Min) de cada una de las variables edáficas para las 150 sub-parcelas de $10 \times 10 \mathrm{~m}$.

Average $(\bar{X} \bar{X})$, standard deviation (S), maximum (Max), and minimum (Min) of each edaphic variable for 150 sub-plots of $10 \times 10 \mathrm{~m}$.

CUADRO 3

ACP y coeficientes de cada una de las variables estructurales y edáficas en una parcela de 6ha (120x500m) en el PNNA usando la BD-1

TABLE 3

PCA and coefficients of each structural and edaphic variable in a 6ha plot (120x500m) in $t$ he Amacayacu National Park using the BD-1

\begin{tabular}{|c|c|c|c|c|c|}
\hline \multirow{11}{*}{ 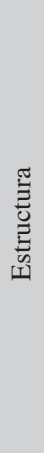 } & Componentes & $\mathrm{C} 1_{\text {est }}$ & $\mathrm{C} 2_{\mathrm{est}}$ & & \\
\hline & Valores propios & 3.07 & 2.54 & & \\
\hline & Varianza $(\%)$ & 43.90 & 36.23 & & \\
\hline & Varianza acumulada $(\%)$ & 43.90 & 80.14 & & \\
\hline & $\mathrm{G}$ & 0.51 & -0.05 & & \\
\hline & Gsot & -0.01 & 0.51 & & \\
\hline & Gdos & 0.51 & -0.10 & & \\
\hline & שֶ & 0.10 & 0.60 & & \\
\hline & Nsot & -0.01 & 0.61 & & \\
\hline & $\mathrm{Ndos}$ & 0.49 & 0.04 & & \\
\hline & $\mathrm{sp}$ & 0.48 & -0.01 & & \\
\hline \multirow{12}{*}{ 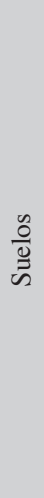 } & Componentes & $\mathrm{C} 1_{\text {edaf }}$ & $\mathrm{C} 2_{\text {edaf }}$ & $\mathrm{C} 3_{\text {edaf }}$ & $\mathrm{C} 4_{\text {edaf }}$ \\
\hline & Valores propios & 2.21 & 2.02 & 1.30 & 1.02 \\
\hline & Varianza $(\%)$ & 27.61 & 25.23 & 16.31 & 12.78 \\
\hline & Varianza acumulada (\%) & 27.61 & 52.85 & 69.15 & 81.93 \\
\hline & $\mathrm{pH}$ & 0.43 & 0.45 & 0.11 & -0.22 \\
\hline & MO & 0.15 & -0.39 & -0.35 & -0.28 \\
\hline & $\underline{\xi}$ & 0.27 & -0.22 & -0.48 & -0.40 \\
\hline & $\stackrel{\overline{0}}{\frac{0}{\pi}}$ & -0.35 & -0.44 & -0.26 & 0.15 \\
\hline & Bases & 0.48 & 0.07 & -0.18 & -0.14 \\
\hline & Arena & 0.09 & -0.43 & 0.59 & -0.34 \\
\hline & Limo & -0.41 & 0.44 & -0.36 & -0.23 \\
\hline & Arcilla & 0.44 & -0.10 & -0.19 & 0.69 \\
\hline
\end{tabular}


correlacionadas de manera negativa y en el cuarto componente la variable más importante es la arcilla ( $\left.\mathrm{C}_{\text {edaf }}\right)$ (Cuadro 3).

Para evaluar la asociación entre variables edáficas y estructurales, se corrieron dos modelos de regresión múltiple entre los valores de los vectores propios de los componentes estructurales del bosque en función de los valores de los vectores propios de los componentes edáficos; ambos modelos fueron significativos ( $\mathrm{p}=0.0273$ y $\mathrm{p}<0.0001$ respectivamente), pero explicaron solo el $7.21 \%$ de la variabilidad en $\mathrm{C} 1_{\text {est }}$ y el $16.60 \%$ en $\mathrm{C} 2_{\text {est }}$. La única variable edáfica estadísticamente significativa en el primer modelo $\left(\mathrm{C}_{\text {est }}\right)$ fue $\mathrm{C} 2_{\text {edaf }}(\mathrm{p}=0.0064)$; las demás variables independientes no fueron significativas $\left(\mathrm{p}=0.7502\right.$ para $\mathrm{C} 1_{\text {edaf }}, \mathrm{p}=0.2513$ para $\mathrm{C} 3_{\text {edaf }}$ y $\mathrm{p}=0.1411$ para $\mathrm{C} 4_{\text {edaf }}$. De manera similar, en el segundo modelo $\left(\mathrm{C}_{\text {est }}\right)$ fueron significativas las variables $\mathrm{C} 1_{\text {edaf }}(\mathrm{p}=0.0234)$, $\mathrm{C} 2_{\text {edaf }}(\mathrm{p}=0.0119)$ y $\mathrm{C} 3_{\text {edaf }}(\mathrm{p}=0.0001)$, mientras que la variable $\mathrm{C}_{\text {edaf }}$ no lo fue $(\mathrm{p}=0.627)$. Con base en estos resultados se eliminaron las variables que no fueron significativas y se corrieron nuevamente las regresiones las cuales explicaron el $4.90 \%$ de la variabilidad en $\mathrm{C}_{1}$ est $(\mathrm{p}=0.0065)$ y el $16.50 \%$ en $\mathrm{C} 2$ est $(\mathrm{p}<0.0001)$ (Cuadro 4). Una exploración visual de los gráficos de los residuales no evidenció una violación del supuesto de normalidad.

El análisis geoestadístico permitió constatar que algunas variables son regionalizadas y se pueden explicar hasta con dos modelos de distribución espacial (lineal, esférico o exponencial), pero aquí sólo se presenta el de mejor ajuste. Para obtener una distribución normal de las variables fue necesaria la transformación de algunas de ellas con funciones matemáticas simples, como logaritmo natural y raíz cuadrada (Hair et al. 2001).

No todas las variables presentaron autocorrelación espacial, entre ellas, las variables estructurales G, Gdos, Ndos y sp, aunque estas dos últimas variables siguen un leve patrón de regionalización por el incremento de la semivarianza con el aumento de la distancia (Fig. 3). Algo similar ocurre con algunas variables edáficas como $\mathrm{pH}, \mathrm{MO}, \mathrm{P}$, bases y arcilla, las cuales presentaron valores de la varianza no explicada $\left(C_{0}\right)$ y de la varianza total $\left(C_{0}+C\right)$ bastante similares (Cuadro 5), aunque sus semivarianzas tienden a aumentar levemente en función de la distancia (Fig. 4).

Al tener en cuenta el modelo más significativo, es decir, aquel con mayor proporción de la varianza explicada por el espacio $\left(C / C_{0}+C\right)$, para Gsot, $\mathrm{N}$ y Nsot, este valor está por encima del 50\% y se presenta autocorrelación espacial a distancias inferiores a 264, 213 y 289m, respectivamente (Cuadro 5). De manera similar, para $\mathrm{Al}$, arena y limo la varianza explicada por el espacio es de 65,73 y $50 \%$ y la autocorrelación espacial tiene lugar a distancias inferiores a 68,421 y $209 \mathrm{~m}$ respectivamente (Cuadro

\section{CUADRO 4}

Regresión múltiple entre los valores de los vectores propios de estructura contra el conjunto de valores de los vectores propios de los componentes edáficos obtenidos en el ACP en una parcela de 6ha (120x500m) en el PNNA

TABLE 4

Multiple regression between each component of forest structure and all significant edaphic components in a 6 ha plot (120x500m) in the Amacayacu National Park

\begin{tabular}{|c|c|c|c|c|c|c|}
\hline Modelo & $\begin{array}{c}\text { Variable } \\
\text { dependiente }\end{array}$ & $\begin{array}{c}\text { Variable } \\
\text { independiente }\end{array}$ & Coeficiente & $\begin{array}{c}\text { Valor-P } \\
\text { (Variable) }\end{array}$ & Razón-F & $\begin{array}{c}\text { Valor-P } \\
\text { (Modelo) }\end{array}$ \\
\hline 1 & $\mathrm{C} 1_{\mathrm{est}}$ & $\mathrm{C} 2_{\text {edaf }}$ & -0.273112 & 0.0065 & 7.62 & 0.0065 \\
\hline \multirow[t]{3}{*}{2} & \multirow[t]{3}{*}{$\mathrm{C} 22_{\text {est }}$} & $\mathrm{C} 1_{\text {edaf }}$ & -0.186131 & 0.0231 & \multirow[t]{3}{*}{9.59} & \multirow[t]{3}{*}{$<0.0001$} \\
\hline & & $\mathrm{C} 2_{\text {edaf }}$ & -0.216579 & 0.0117 & & \\
\hline & & $\mathrm{C} 3_{\text {edaf }}$ & 0.434396 & 0.0001 & & \\
\hline
\end{tabular}

El primer modelo explica sólo el $4.90 \%$ de la variabilidad en $\mathrm{C}_{\mathrm{est}}$ y el segundo el $16.50 \%$ de la variabilidad en $\mathrm{C} 22_{\mathrm{est}}$. The first model explains only $4.90 \%$ of variability of $\mathrm{C}_{1}$ est , and the second model explains $16.50 \%$ of variability of $\mathrm{C} 2{ }_{\text {est }}$. 
G

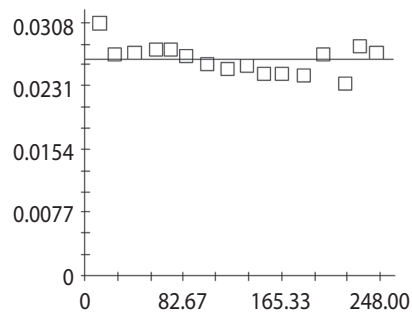

Gsot

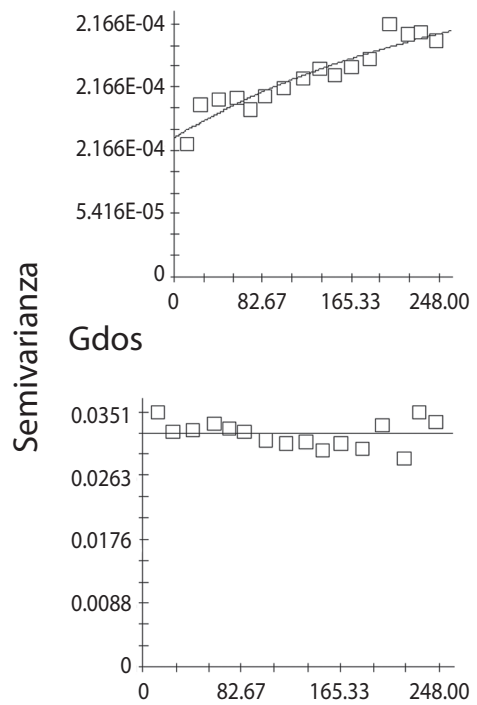

N

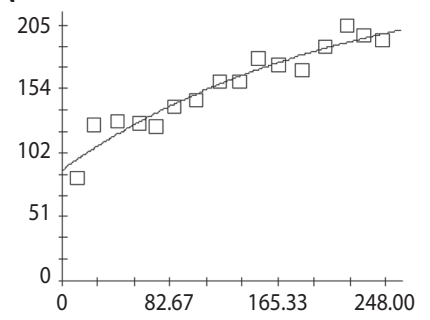

Nsot

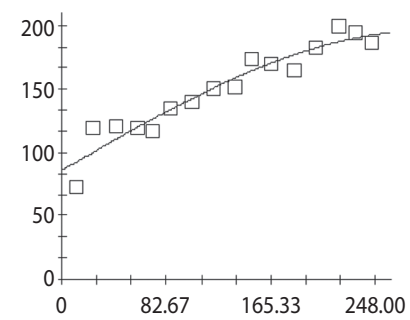

Ndos

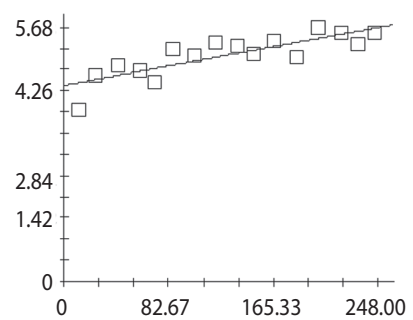

$\mathrm{Sp}$

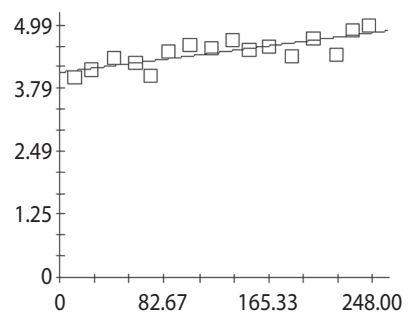

\section{Distancia}

Fig. 3. Variogramas isotrópicos de las variables estructurales en una parcela de 6 ha $(120 \times 500 \mathrm{~m})$ en el PNNA usando la BD-2. El eje de las abcisas representa la distancia en metros y el eje de las ordenadas representa la semivarianza. G: modelo lineal. Gsot: modelo exponencial. Gdos: modelo lineal. N: modelo exponencial. Nsot: modelo esférico. Ndos: modelo lineal. Sp: modelo lineal.

Fig. 3. Isotropic variograms of structural variables in a 6-ha plot (120x500m) in the Amacayacu National Park using the BD-2. X-axis represents the distance in meters and Y-axis represents the semivariance. G: linear model. Gsot: exponential model. Gdos: linear model. N: exponential model. Nsot: espheric model. Ndos: linear model. sp: linear model. 
$\mathrm{pH}$

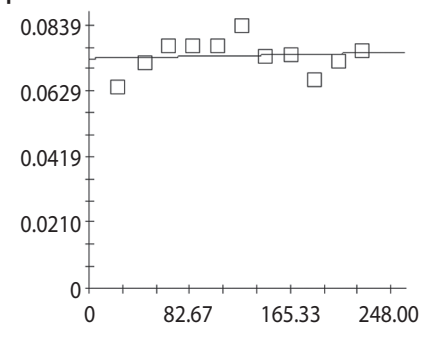

MO

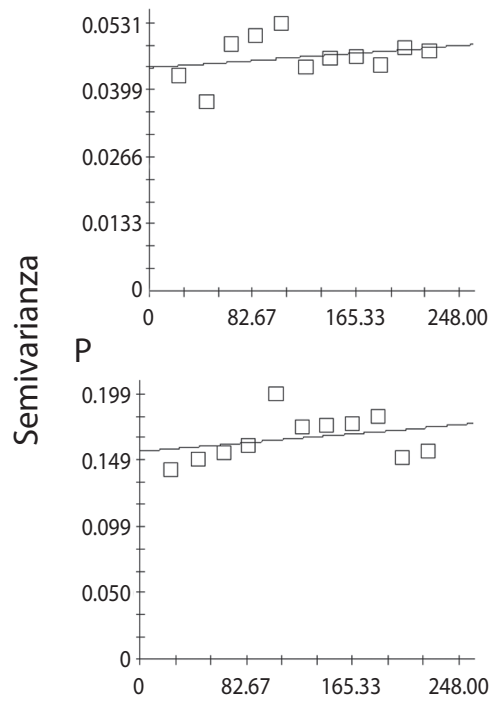

\section{Al}

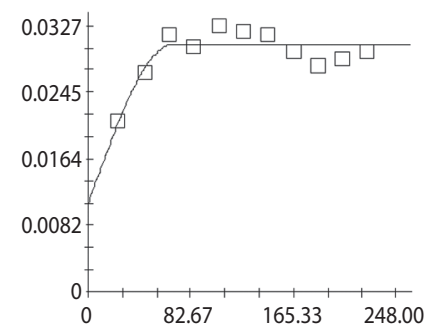

Bases

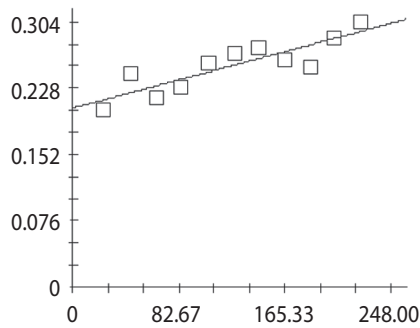

Arena

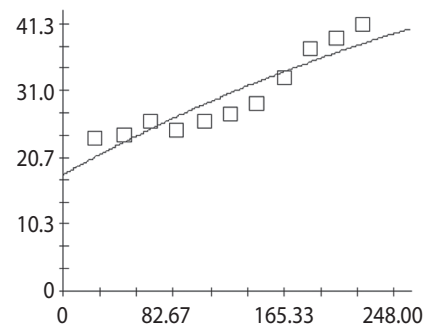

Limo

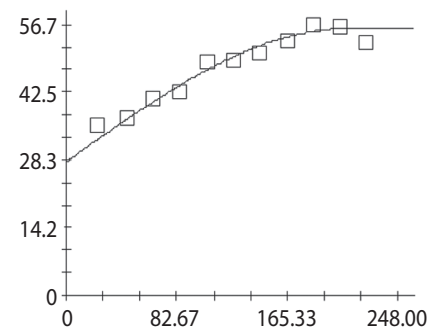

Arcilla

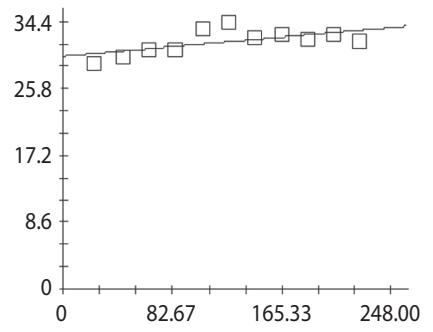

\section{Distancia}

Fig. 4. Variogramas isotrópicos de las variables edáficas en una parcela de 6ha (120x500m) en el PNNA usando la BD-1. El eje de las abcisas representa la distancia en metros y el eje de las ordenadas representa la semivarianza. pH: modelo lineal. MO: modelo lineal. P: modelo lineal. Al: modelo esférico. Bases: modelo lineal. Arena: modelo exponencial. Limo: modelo esférico. Arcilla: modelo lineal.

Fig. 4. Isotropic variograms of edaphic variables in a 6ha plot (120x500m) in the Amacayacu National Park using the BD-1. $\mathrm{X}$-axis represents the distance in meters and Y-axis represents the semivariance. pH: linear model. Organic matter (MO): linear model. P: linear model. Al: espherical model. Soil cations: linear model. Sand: exponential model. Silt: espherical model. Clay: linear model. 
CUADRO 5

Análisis geoestadístico de las variables estructurales (BD-2) y edáficas (BD-1) en una parcela de 6ha (120x500m) en el PNNA

TABLE 5

Geostatistical analysis of structural (BD-2) and edaphic (BD-1) variables in a 6ha plot

(120x500m) in the Amacayacu National Park

\begin{tabular}{|c|c|c|c|c|c|c|}
\hline Variable & Modelo & $C_{0}$ & $C_{0}+C$ & $A_{0}$ & $C / C_{0}+C$ & $\mathrm{R}$ \\
\hline $\mathrm{G}^{1}$ & Lineal & 0.02639 & 0.02639 & 244.93 & 0 & 0 \\
\hline Gsot & Exponencial & 0.00012 & 0.00027 & 264.70 & 56 & 88 \\
\hline $\operatorname{Gdos}^{1}$ & Lineal & 0.03204 & 0.03204 & 244.93 & 0 & 0 \\
\hline $\mathrm{N}$ & Exponencial & 88.60 & 248.30 & 213.60 & 64 & 92 \\
\hline Nsot & Esférico & 86.00 & 196.70 & 289.00 & 56 & 92 \\
\hline Ndos & Lineal & 437.931 & 56.628 & 244.93 & 23 & 68 \\
\hline $\mathrm{sp}$ & Lineal & 404.450 & 48.309 & 244.93 & 16 & 6 \\
\hline $\mathrm{pH}$ & Lineal & 0.07307 & 0.07488 & 224.16 & 2 & 1 \\
\hline $\mathrm{MO}^{2}$ & Lineal & 0.04419 & 0.04816 & 224.16 & 8 & 8 \\
\hline $\mathrm{P}^{2}$ & Lineal & 0.15516 & 0.17318 & 224.16 & 10 & 10 \\
\hline $\mathrm{Al}$ & Esférico & 0.01063 & 0.03036 & 68.60 & 65 & 75 \\
\hline Bases $^{2}$ & Lineal & 0.20463 & 0.29256 & 224.16 & 30 & 76 \\
\hline Arena & Exponencial & 18 & 67 & 421.40 & 73 & 85 \\
\hline Limo & Esférico & 27.89 & 55.79 & 209.5 & 50 & 96 \\
\hline Arcilla & Lineal & 2.992 .311 & 3.324 .876 & 224.16 & 10 & 36 \\
\hline
\end{tabular}

5). Lo anterior significa que cada variable es regionalizada dentro de su rango pero más allá de esta distancia la variable es independiente.

En los mapas de la Fig. 5 se esquematizan algunas variables estructurales que presentaron una distribución espacial claramente definida como Gsot, N y Nsot; los valores más bajos de estas variables se presentaron en el extremo norte de la parcela y, tanto la densidad total como la de individuos de sotobosque se concentraron en el centro, con disminuciones graduales hacia el sur y más marcadas hacia el norte; de hecho, en el extremo nororiental de la parcela parece existir menor densidad total y de individuos de sotobosque. Por otra parte, los contenidos de limo y arena son opuestos entre sí, pues ésta última variable parece estar en mayor cantidad en el sector suroccidental y en menor proporción en el norte, mientras que para el limo la distribución es inversa; finalmente, en el centro de la parcela parece haber mayor concentración de $\mathrm{Al}$ en el suelo (Fig. 6).

\section{DISCUSIÓN}

Asociación entre variables edáficas y estructura del bosque: El mayor número de individuos en las clases diamétricas inferiores encontrado en el presente estudio concuerda con las características reportadas en la literatura para los bosques tropicales primarios (Baumgartner 1980), lo que significa que los individuos del sotobosque representan un alto porcentaje de la densidad del bosque. Otros componentes de la estructura como el área basal por hectárea, muestran un comportamiento 

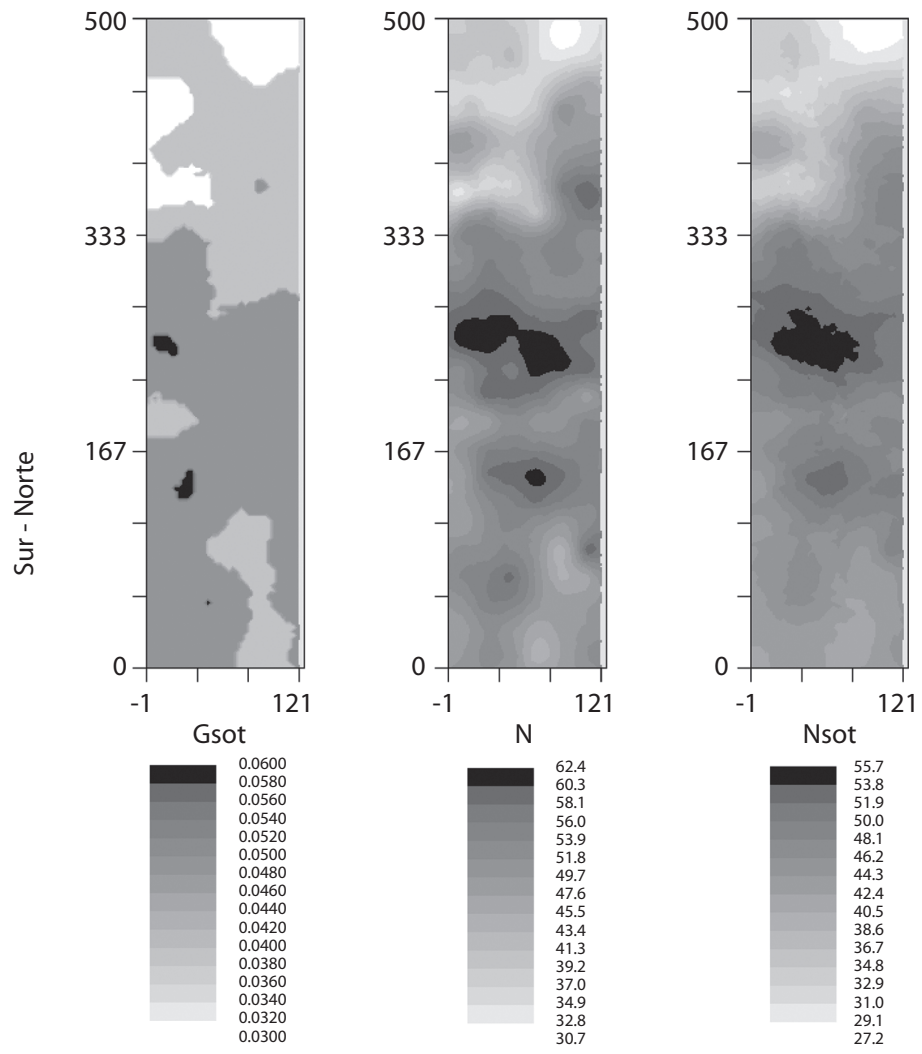

Occidente - Oriente

Fig. 5. Distribución espacial de las variables estructurales que presentaron autocorrelación espacial realizado por interpolación con el método krigging para una parcela de 6ha (120x500m) en el PNNA usando la BD-2.

Fig. 5. Spatial distribution of structural varibales based on krigging interpolation in a 6ha plot (120x500m) in the Amacayacu National Park using the BD-2.

opuesto, pues el mayor aporte lo hacen los individuos de las clases diamétricas superiores, lo cual también concuerda con otros estudios (Williams-Linera 1990). Esto significa que si bien, el sotobosque es numéricamente importante, el espacio de crecimiento es ocupado principalmente por los árboles del dosel.

Por otra parte, los resultados sobre el análisis de las relaciones entre estructura del bosque y suelos sugieren que la estructura del dosel está asociada de manera negativa con un grupo de variables edáficas compuesto por $\mathrm{pH}$ y limo, y de manera positiva con arena y $\mathrm{Al}$ (segundo componente edáfico). Esto quiere decir, que en aquellos lugares donde hay valores mayores de $\mathrm{pH}$ y bajos contenidos de arena la estructura de los individuos del dosel es menos compleja (es decir, con menor área basal, número de individuos y número de especies); en otras palabras, la estructura del dosel es más compleja en lugares de alta acidez y posiblemente buen drenaje. También, se encontró que existe una asociación negativa entre la estructura de los individuos del sotobosque y dos grupos de variables edáficas que tal vez estén aludiendo al componente químico del suelo $\left(\mathrm{Cl}_{\text {edaf }}\right.$ y $\left.\mathrm{C} 2_{\text {edaf }}\right)$ y una correlación positiva con otro grupo asociado al drenaje ( $\mathrm{C} 3_{\text {edaf }}$ ); esto significa que en los lugares 

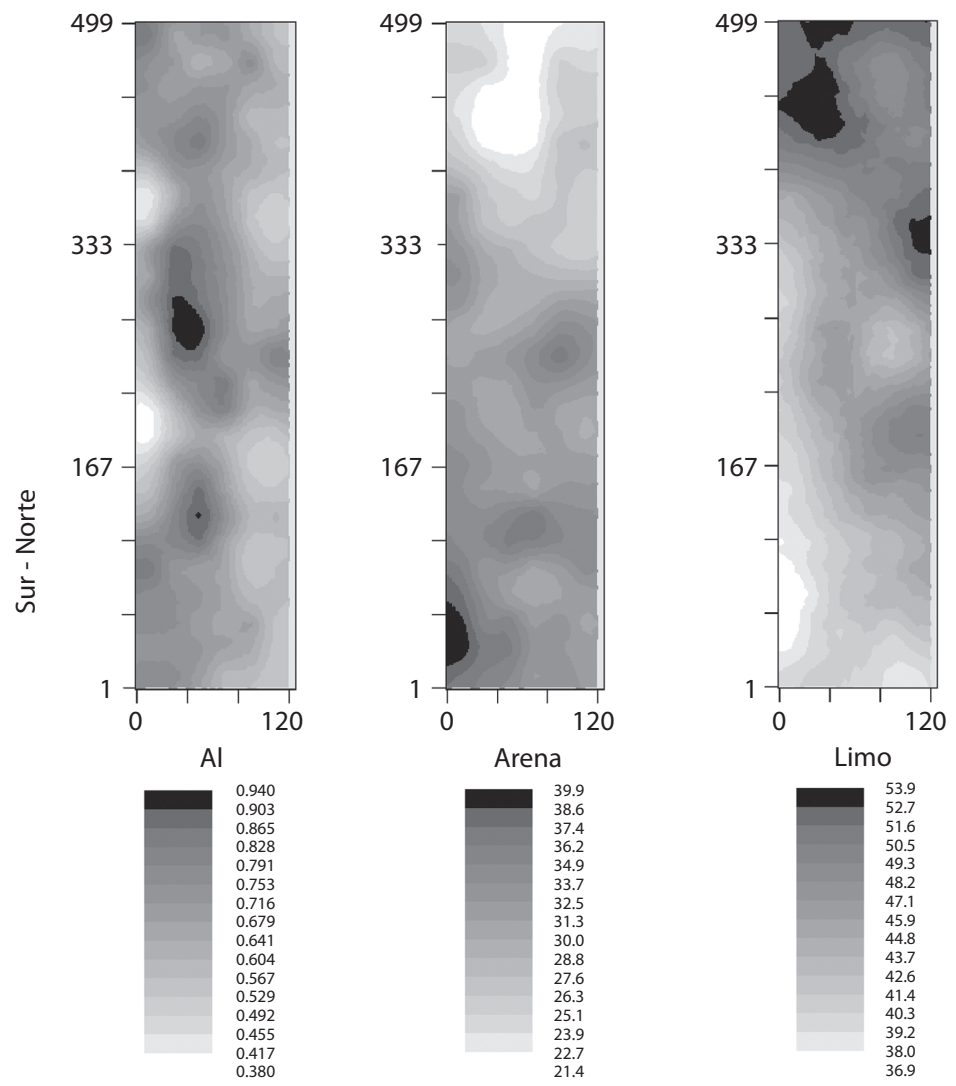

Occidente - Oriente

Fig. 6. Distribución espacial de las variables edáficas que presentaron autocorrelación espacial realizado por interpolación con el método krigging para una parcela de 6ha (120x500m) en el PNNA usando la BD-1.

Fig. 6. Spatial distribution of edaphic varibales based on krigging interpolation in a 6ha plot (120x500m) in the Amacayacu National Park using the BD-1.

donde hay menor contenido de bases asociado a valores bajos de $\mathrm{pH}$ pero altos de $\mathrm{Al}$ y arena, la estructura del sotobosque es más compleja. De este modo, los resultados encontrados, tanto para la estructura del dosel como del sotobosque, tienen en común su asociación con un mismo grupo de variables edáficas $\left(\mathrm{C} 2_{\text {edaf }}\right)$, de tal manera que en el PNNA estructuras más complejas de la vegetación se presentan en sitios con suelos más ácidos y con mayor contenido de arena.

Los resultados de Duivenvoorden et al. (2005) son similares a los de este estudio; ellos también encontraron en bosques de la
Amazonía asociaciones negativas entre la densidad de individuos y las bases del suelo y argumentaron que esto posiblemente se debía a que los árboles grandes se establecían en sitios con mayor disponibilidad de bases, y los mismos imponían restricciones a la entrada de luz para los individuos del sotobosque, lo cual resulta en menores densidades; sin embargo, los resultados de este estudio no evidencian asociaciones entre los individuos del dosel y las bases del suelo por lo que esta explicación no resulta apropiada en este caso. Tal vez las restricciones de luz por parte de los árboles grandes sí 
pueden estar ocurriendo dentro de la parcela, pero ello no se evaluó en el presente estudio.

Las arcillas están cargadas eléctricamente y son las principales responsables de la capacidad de intercambio de cationes del suelo, por lo que es de esperarse que a ellas estén asociados valores altos de $\mathrm{pH}$ y de bases intercambiables (primer componente edáfico, Cuadro 3); así mismo, se espera que en los sitios con altos contenidos de arcilla la velocidad con la cual pasa el agua al interior del suelo sea lenta a causa de la baja porosidad (Jaramillo 2002), lo que puede provocar encharcamientos, cuando se presentan eventos de lluvia. A su vez, la respiración de las raíces de las plantas se puede ver limitada bajo tales circunstancias debido a estrés por anoxia, lo cual limitaría el establecimiento de individuos arbóreos en tales sitios (Duivenvoorden 1996); de este modo, presentarían una menor densidad de individuos. Así, los árboles que se establezcan en lugares encharcados deberían estar adaptados fisiológicamente a dichas condiciones (Wittmann et al. 2004).

Clark \& Clark (2000) encontraron en suelos fértiles de Costa Rica procesos similares a los explicados arriba, en los cuales la densidad de individuos era menor en suelos fértiles debido a que estos sitios presentaban inundaciones periódicas. Los resultados de este estudio muestran que probablemente el factor edáfico clave para explicar las variaciones estructurales, tanto en el dosel como en el sotobosque, es el contenido de arena. En el sotobosque el efecto de la arena pareciera ser el más importante, debido a que todos los coeficientes de esta variable fueron altos tanto en el componente edáfico dos como en el tres, los cuales se correlacionaron de manera significativa con la estructura del sotobosque; este resultado se explica en virtud de que el contenido de arena puede desencadenar una serie de procesos importantes para el desarrollo de las plantas, entre ellos los relacionados con el drenaje y la aireación del suelo.

Así pues, el análisis de regresión mostró que la estructura del sotobosque se asocia de manera positiva con el tercer componente edáfico, en el cual el fósforo presenta un alto coeficiente negativo y la arena un coeficiente positivo; entonces, estructuras más complejas del sotobosque están presentes en sitios con mayor contenido de arena pero más bajo de fósforo. Aunque se ha documentado que en los bosques tropicales de tierras bajas el fósforo es escaso y por lo tanto suele ser un factor limitante para el desarrollo de estructuras exuberantes de la vegetación (Vitousek 1984). La cantidad de fósforo disponible en la matriz del suelo puede no ser crucial debido a que las plantas han desarrollado mecanismos muy eficientes de conservación de este nutriente. En consecuencia, generalmente a través de la hojarasca no retornan al suelo grandes cantidades de fósforo; al contrario, la mayoría de las plantas lo transfieren eficientemente de las hojas senescentes a hojas mas nuevas (Montagnini \& Jordan 2002), pues este es un nutriente con alta movilidad en la planta (Salisbury \& Ross 1994).

Estructuras menos complejas del sotobosque se presentan en lugares con alto contenido de fósforo donde a su vez hay bajo contenido de arena y por ende se espera que el drenaje sea lento. Como se ha explicado atrás, estos resultados sugieren que el desarrollo estructural del sotobosque depende poco de la disponibilidad de fósforo y más bien depende del contenido de arena en el suelo. En consecuencia, la correlación negativa entre estructura del sotobosque y contenido de fósforo del suelo probablemente no significa una relación causa-efecto sino más bien circunstancial, asociada con el drenaje lento en sitios pobres en arena (y ricos en arcilla y limo) donde el fósforo es más abundante. Estos resultados también sugieren que los mecanismos de conservación de nutrientes en esta parcela tal vez son eficientes, asunto que ha sido observado por Vitousek \& Sanford (1986) al estudiar los ciclos de nutrientes en algunos bosques tropicales de tierras bajas.

Análisis espacial de las variables edáficas y estructurales del bosque: Según los resultados de este análisis espacial, las cuatro variables incluidas en el análisis de la estructura del dosel tienen distribución aleatoria en 
el espacio a la escala evaluada, mientras que las variables concernientes a la estructura del sotobosque presentan autocorrelación espacial. Wagner (2003) menciona tres causas que pueden explicar la autocorrelación espacial de estas variables: 1) los mecanismos de dispersión, 2) las relaciones interespecíficas y 3) la respuesta a factores ambientales, los cuales también están espacialmente estructurados. Aunque los análisis de este estudio no pueden evaluar formalmente ninguno de los tres argumentos propuestos por este autor, hay razones para creer que la densidad de individuos puede estar relacionada con la distribución de arena en el espacio (tercera causa).

He et al. (1996) evaluaron la distribución espacial de la riqueza y el número de individuos en bosques de Malasia y encontraron que la primera variable era aleatoria, mientras que la segunda sí presentó autocorrelación espacial en un rango aproximado de $400 \mathrm{~m}$. Los autores concluyeron que en esos bosques tal vez ocurrían procesos de diferenciación de nicho a microescala $(<20 \mathrm{~m})$ debido al alto valor de la varianza no explicada por el modelo (nugget o efecto pepita) observado en el variograma de riqueza. Este trabajo presenta resultados similares (riqueza aleatoria y densidad de individuos estructurada en el espacio); sin embargo, no hay evidencias que permitan afirmar con certeza que en el PNNA estén ocurriendo procesos de diferenciación de nicho, incluso en escalas inferiores a la evaluada por este estudio $(<10 \mathrm{~m})$. Entre tanto, la variación de la densidad de individuos en la parcela parece estar respondiendo a un gradiente espacial relacionado con la arena: al hacer una inspección visual de los mapas de distribución espacial de la densidad de individuos total y de sotobosque, y de la distribución espacial de arena se puede notar que hay correspondencia entre los valores altos y bajos de estas variables. Según se expresó anteriormente, el contenido de arena puede desencadenar una cascada de características y procesos que afectan el desarrollo de la vegetación, entre ellas mayor porosidad del suelo y drenaje más rápido (Jaramillo 2002). Probablemente la topografía de la parcela está relacionada con la distribución de la fracción arena y arcilla del suelo, por lo cual sería interesante incluirla en la evaluación.

En síntesis, en la parcela del PNNA parece existir una asociación entre la estructura del bosque (con mayor fuerza la referente al sotobosque) y el contenido de arena, el cual condiciona el drenaje y la aireación del suelo. Aunque no se puede afirmar con certeza que la ubicación de la arena en el espacio esté condicionando la densidad de individuos, se mostró que esto puede ser posible. La mayoría de las variables edáficas de este estudio (excepto arena, limo y aluminio) mostraron una distribución aleatoria en el espacio y quizá se deba a que la formación de los suelos del PNNA deriva de una misma unidad geológica llamada Formación Pebas o Solimoes (Herrera 1997), lo que sugiere que los procesos de formación de suelo en este lugar son homogéneos y en consecuencia, que las arcillas, el pH y las bases no muestren una estructura espacial en la escala trabajada.

\section{AGRADECIMIENTOS}

Este trabajo fue realizado en el marco del proyecto Estructura, Diversidad y Dinámica de Especies Arbóreas en Bosques de Tierra Firme de la Amazonía Colombiana financiado por COLCIENCIAS (CT 1118-333-18676) y el Center for Tropical Forest Science - CTFS (subcontrato No. 08-440-0000137735) y ejecutado por la Universidad Nacional de Colombia Sede Medellín. Agradecemos al Instituto Amazónico de Investigaciones Científicas SINCHI y a la Unidad Administrativa Especial del Sistema de Parques Nacionales Naturales de Colombia, en especial a los funcionarios y contratistas del Parque Nacional Natural Amacayacu. Agradecemos de manera especial a Álvaro Javier Duque, Kenneth Roy Cabrera, Daniel Jaramillo, Álvaro Lema Tapias y Luisa Fernanda Casas por sus valiosos aportes en diferentes fases de este trabajo, así como al grupo de estudiantes de Ingeniería Forestal de la Universidad Nacional y a la comunidad indígena 
Tikuna de Palmeras, por su colaboración en el trabajo de campo.

\section{RESUMEN}

Las diferencias estructurales del bosque pueden ser el producto de variaciones ambientales a diferentes escalas, entre ellas los suelos. Para evaluar tal asociación, se usaron los datos de estructura del bosque y de suelos de una parcela de 6ha establecida en un bosque de tierra firme de la Amazonía colombiana. Las variables edáficas y estructurales se redujeron con un análisis de componentes principales (ACP) y un análisis de regresión múltiple verificó si existía asociación entre los componentes edáficos y estructurales del ACP. La regionalización de las variables de estudio se verificó con variogramas isotrópicos; además, se construyeron mapas de distribución espacial. El ACP de las variables estructurales identificó dos componentes: dosel y sotobosque, los cuales explicaron el 43.9 y $36.2 \%$ de la varianza total, respectivamente; para las variables edáficas los cuatro primeros componentes explicaron globalmente el $81.9 \%$ de la varianza total. Los análisis de regresión mostraron que tanto el dosel como el sotobosque se asocian con mayores contenidos de arena y baja fertilidad del suelo $(\mathrm{p}<0.05)$, pero explicaron una proporción baja de la variabilidad total $\left(\mathrm{R}^{2}=4.9 \%\right.$ para dosel y $16.5 \%$ para sotobosque). Las tres variables de la estructura del sotobosque, Al, limo y arena presentaron autocorrelación espacial.

Palabras clave: área basal, autocorrelación espacial, densidad de individuos, Parque Nacional Natural Amacayacu, variables edáficas.

\section{REFERENCIAS}

Barreto, J., A. Duque, D. Cárdenas \& F. Moreno. 2010. Variación florística de especies arbóreas a escala local en un bosque de tierra firme en la Amazonia colombiana. Acta Amazonica 40: 179-188.

Baumgartner, A. 1980. El bosque tropical y la biosfera, p. 36-67. In UNESCO/CIFCA (eds.). Ecosistemas de los bosques tropicales: informe sobre el estado de conocimientos. Serie de investigaciones sobre los recursos naturales, XVI. Madrid, España.

Camarero, J.J. \& E. Gutiérrez. 1999. Patrón espacial de un ecotono bosque subalpino-pastos alpinos (las cutas, ordesa, pirineos centrales). Invest Agr: Sist Recur For 8: 171-205.

Chamorro, C. 1989. Biología de los suelos del Parque Nacional Natural Amacayacu, y zonas adyacentes (Amazonas, Colombia). Colombia Geográfica 15: 45-63.
Clark, D.B. 2002. Los factores edáficos y la distribución de las plantas, p. 193-221. In M.R. Guariguata \& G.H. Kattan (eds.). Ecología y conservación de bosques neotropicales. LUR, Cartago, Costa Rica.

Clark, D.B. \& D.A. Clark. 2000. Landscape-scale variation in forest structure and biomass in a tropical rain forest. Forest Ecol. Manag. 137: 185-198.

Duivenvoorden, J.F. 1996. Patterns of tree species richness in rain forest of the middle Caquetá area, Colombia, NW, Amazonia. Biotropica 28: 142-158.

Duivenvoorden, J.F., A. Duque, J. Cavelier, A. García, C. Grandez, M.J. Macía, H. Romero-Saltos, M. Sánchez \& R. Valencia. 2005. Density and diversity of plants in relation to soil nutrient reserves in well-drained upland forests in the north-western Amazonian basin. Biol. Skr. 55:25-35.

Gallardo, A. 2006. Geostadística. Ecosistemas 15: 48-58.

Gilbert, B. \& M.J. Lechowicz. 2004. Neutrality, niches, and dispersal in a temperate forest understory. PNAS 101: 7651-7656.

Giraldo H.R., W. Troncoso, J.E. Mancera \& N. Méndez. 2000. Geoestadística: una herramienta para la modelación en estuarios. Rev Acad Colomb Cienc 24: 59-72.

Guo, D., P. Mou, R.H. Jones \& R.J. Mitchell. 2002. Temporal changes in spatial patterns of soil moisture following disturbance: an experimental approach. J. Ecol. 90: 338-347.

Hair, J.F., Jr. R.E. Anderson, R.L. Tatham \& W.C. Black. 2001. Análisis Multivariante. Prentice Hall, México, D.F., México.

Hartshorn, G.S. 2002. Biogeografía de los bosques neotropicales, p. 59-81. In M.R. Guariguata \& G.H. Kattan (eds.). Ecología y conservación de bosques neotropicales. LUR, Cartago, Costa Rica.

He, F., P. Legendre \& J.V. LaFrankie. 1996. Spatial pattern of diversity in a tropical rain forest in Malaysia. J. Biogeography 23: 57-74.

Herrera, J. 1997. Geografía, p. 137-163. In Instituto Geográfico Agustín Codazzi-IGAC (ed.). Zonificación ambiental para el plan modelo Colombo-Brasilero (Eje Apaporis-Tabatinga: PAT). IGAC, Bogotá, Colombia.

Jackson, R.B. \& M.M. Caldwell. 1993. The scale of nutrient heterogeneity around individual plants and its quantification with geostatistics. Ecology 74: 612-614. 
Jaramillo J.D. 2002. Introducción a la ciencia del suelo. Investigación (Geociencias). Universidad Nacional de Colombia Sede Medellín, Medellín, Colombia.

Montagnini, F. \& C. Jordan. 2002. Reciclaje de nutrientes, p. 167-191. In M.R. Guariguata \& G.H. Kattan (eds.). Ecología y conservación de bosques neotropicales. LUR, Cartago, Costa Rica.

Rudas, A. \& A. Prieto. 1998. Análisis florístico del Parque Nacional Natural Amacayacu e Isla de Mocagua, Amazonas (Colombia). Caldasia 20: 142-172.

Salisbury, F.B. \& C.W. Ross. 1994. Fisiología Vegetal. Iberoamérica, México D.F., México.

Scatena, F.N. 2002. El bosque neotropical desde una perspectiva jerárquica, p. 23-41. In M.R. Guariguata \& G.H. Kattan (eds.). Ecología y conservación de bosques neotropicales. LUR, Cartago, Costa Rica.

Schwars, P.A., T.J. Fahey \& C.E. McCulloch. 2003. Factors controlling spatial variation of trees species abundance in a forested landscape. Ecology 84: 1862-1878.

Sierra, U.C.A. 2001. Biomasa de raíces en bosques secundarios y primarios del área de influencia de la central hidroeléctrica Porce II. Tesis Ingeniería Forestal, Universidad Nacional de Colombia Sede Medellín, Medellín, Colombia.

Soares-Filho, B.S., D.C. Nepstad, L.M. Curran, G.C. Cerqueira, R.A. Garcia, C.A. Ramos, E. Voll, A. McDonald, P. Lefebvre \& P. Schlesigner. 2006.
Modelling conservation in the Amazon basin. Nature 440: 520-523.

Svenning, J.C. 1999. Microhabitat specialization in a species-rich palm community in Amazonian Ecuador. J. Ecol. 87: 55-65.

Vitousek, P.M. 1984. Litterfall, nutrient, cycling and nutrient limitation in tropical forest. Ecology 65: 285-298.

Vitousek, P.M. \& R.L. Sanford. 1986. Nutrient cycling in moist tropical forest. Annu. Rev. Ecol. Syst. 17: 137-167.

Wagner, H.H. 2003. Spatial covariance in plant communities: integrating ordination, geostatistics, and variance testing. Ecology 84: 1045-1057.

Wagner, H.H. \& M.J. Fortin. 2005. Spatial analysis of landscapes: concepts and statistics. Ecology 86: 1975-1987.

Williams-Linera, G. 1990. Vegetation structure and environmental conditions of forest edges in Panama. J. Ecol. 78: 356-373.

Wittmann, F., W.J. Junk \& M. Piedade. 2004. The várzea forests in Amazonia: flooding and the highly dynamic geomorphology interact with natural forest succession. Forest Ecol. Manag. 196: 199-212.

FAO. 2009. Situación de los bosques del mundo 2001. FAO, Roma, Italia. (Consultado: Octubre 19, 2009, www. fao.org/docrep/003/y0900s/y0900s05.htm\#P0_0). 